\title{
«Oft fehlt die Zivilcourage»
}

\section{Daniel Lüthi}

Freier Journalist und Fotograf, Medientrainer, Bern

Der Jahreswechsel ist eine Zeit des Innehaltens. Man schaut zurück und nach vorne, analysiert, meditiert, sucht Verbesserungsmöglichkeiten. Bei Mechtild Willi passt die stille und besinnliche Zeit dieses Jahr besonders gut zur aktuellen Lebensphase: Bis Ende Januar noch befindet sie sich in einem sechsmonatigen Sabbatical, einer Auszeit, die sie auch für die selbstkritische Reflexion nutzt: «Wer bin ich ohne meine Arbeit?», frage ich mich zum Beispiel.»

\section{Selbstgewählt - oder nicht}

Mechtild Willi hat diesen Zwischenhalt, diesen Unterbruch selber gewählt. Er ist auch ein Privileg nach langjährigem Arbeitseinsatz: "Mir wurde bewusst, dass meine letzte berufliche Phase beginnt. Und da ich diesbezüglich einen fortschrittlichen Arbeitgeber habe, der eine solche Pause ermöglicht, wollte ich mir ein paar Fragen stellen. Dabei versuche ich, auch eigenem Unvermögen auf die Spur zu kommen.» Konkreter: «Ich bin eine umtriebige Person, liebe den Diskurs und die Irritation. Manchmal überfordere ich damit wohl mein Umfeld.»

Die Leute, für die sie in ihrem beruflichen Alltag arbeitet, trifft der Moment, in dem die Zeit still zu stehen scheint, in aller Regel völlig unerwartet. Es ist ein Schicksalsschlag, der sie zum Innehalten zwingt, oft ein Unfall, der ihren Körper - und bisweilen wohl auch ihren Lebenswillen - lähmt, sie von Fussgängern zu Rollstuhlfahrern macht, zu Para- oder Tetraplegikern. Von einer Sekunde auf die andere. Zusammen mit zwei Betroffenen hat Willi ein Buch herausgegeben mit dem Titel: «Warum das Leben weitergeht» (vgl. Hinweis), trotzdem weitergeht, tönt

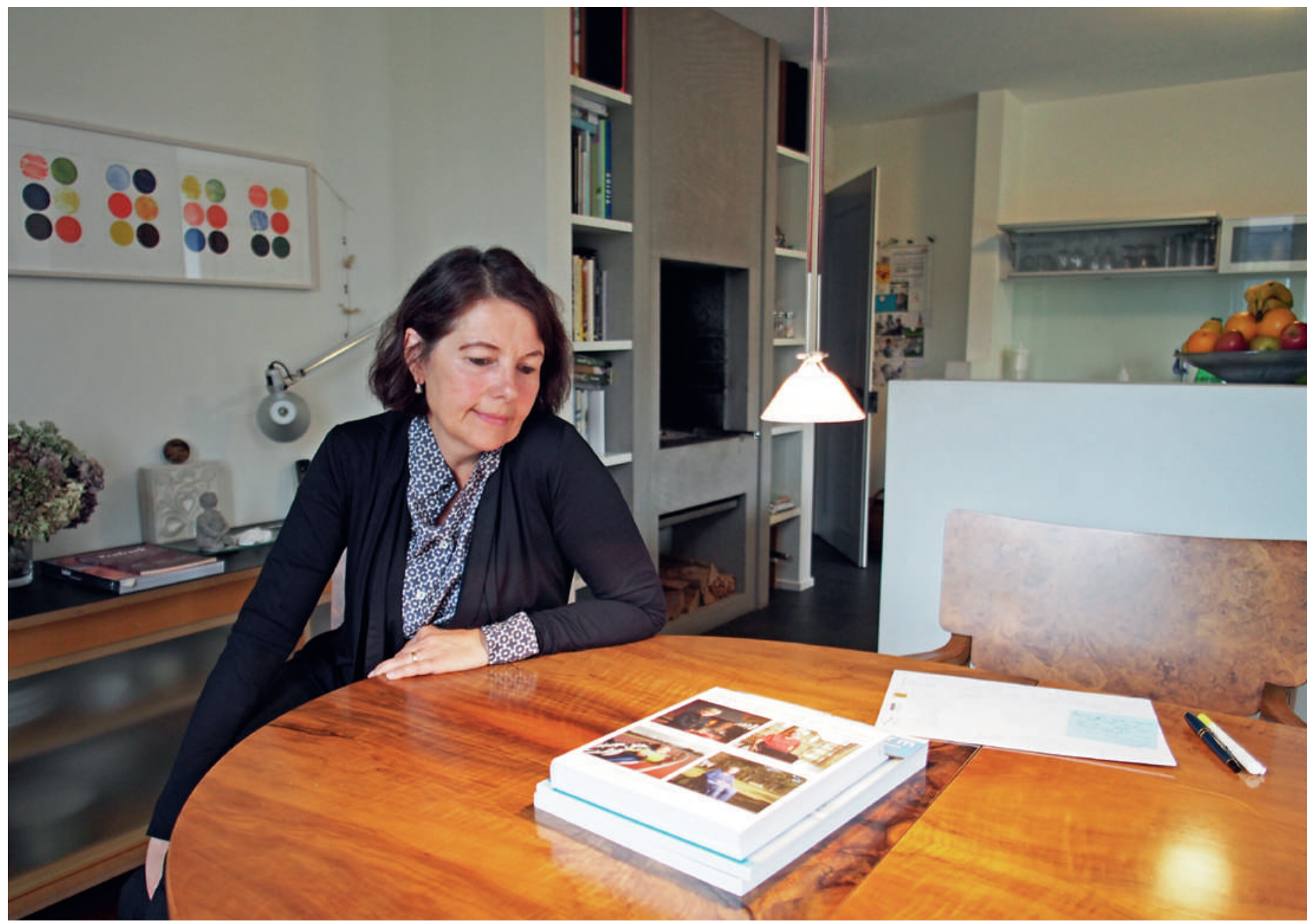


mit, «auch im Alter und mit Behinderung», wie es im Untertitel heisst. Autonomie, Würde und Lebendigkeit sind Themen, und der Erfahrungsvorsprung von Menschen mit einer Behinderung. "Wir alle, die kein solches Schicksal haben, kennen unsere eigenen Ressourcen nicht", kommentiert Willi, "der Mensch kann in solchen Situationen einiges mobilisieren. Andere kommen an diese Quelle gar nie heran.»

\section{Medizinische Maschinerie}

Sie selber habe so etwas ja nie erlebt, fügt sie hinzu, "wir Profis können nur aus der Erfahrung sprechen, die wir in einer solchen Situation mit anderen gemacht haben.» Was für sie selber nur bedingt stimmt, wie sich herausstellt. Mechtild Willi hat sich nämlich intensiv mit der Frage einer Behinderung beschäftigt, und sie spricht in aller Offenheit auch darüber. «Ich habe spät geheiratet und wurde spät schwanger», erzählt sie, im Alter von 42 Jahren erwartete sie ihr erstes Kind, ein Risikotest ergab eine hohe Wahrscheinlichkeit, dass dieses mit einer Trisomie 21, also einem Down-Syndrom auf die Welt kommen würde. «Ungewollt geriet ich in eine medizinische Maschinerie, der ich persönlich sehr kritisch gegenüberstehe.» Ein Chefarzt des Spitals, in dem sie damals

"Wir alle, die kein solches Schicksal haben, kennen unsere eigenen Ressourcen nicht.»

arbeitete, habe sie angehört und beraten. «Ich müsse mir selber die Frage stellen, sagte er, ob eine solche Behinderung unseres Kindes für mich ein Schicksal wäre, das ich tragen könne, oder aber einen Lebensbruch bedeuten würde. Mein Berufsleben, das mir sehr viel bedeutet, stand auf dem Spiel.» Nach weiteren Abklärungen erübrigte dann ein Brief im Briefkasten die Antwort auf die Frage des Chefarztes - er enthielt die befreiende Nachricht: Beim Kind war alles gut.

\section{Was ist sinnvoll?}

Eine weitere Frage beschäftigt Mechtild Willi seither fast mehr: Ist alles, was medizinisch machbar ist, auch sinnvoll? "Ich denke, dass es zwei Schienen braucht», antwortet sie selber. "Es ist richtig, dass intensiv geforscht wird, wie Nervenzellen regeneriert werden könnten. Wenn wir etwas finden, was Behinderten querschnittgelähmten Menschen helfen kann, umso besser. Innovation aber um jeden Preis finde ich falsch. Denn gleichzeitig müssen wir uns damit beschäftigen, wie wir auch mit einer Behinderung oder mit Einschränkungen gesund und erfüllt

\section{Mechtild Willi Studer}

Mechtild Willi Studer wurde 1959 in Baden geboren. Die Schulen besuchte sie in Killwangen und Boswil (AG), dann arbeitete sie als Schwesternhilfe im Kreisspital Muri (AG). In Rheinau absolvierte sie 1978 bis 1981 die Ausbildung in psychiatrischer Krankenpflege. 1982 bis 1992 arbeitete sie an der Psychiatrischen Universitätsklinik

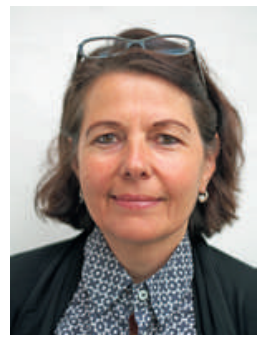

Burghölzli in Zürich. 1993 bis 1997 war sie Geschäftsleiterin des Spitex-Verbandes des Kantons Zürich, dann bis 2004 Pflegedienstleiterin im Bezirksspital Affoltern am Albis. Seit 2004 ist sie Leiterin Pflegemanagement im Schweizer Paraplegiker-Zentrum in Nottwil. Mechtild Willi ist verheiratet und Mutter eines 14-jährigen Sohnes. Sie wohnt mit ihrer Familie in Knonau.

leben können. Es geht auch um anderes als um das Reparieren und Wiederherstellen.» Der Film «Le huitième jour" kommt ihr in den Sinn: "Hier findet ein Manager dank eines Mongoloiden die Essenz des Lebens.»

Ausdrücklich erwähnt sie in diesem Zusammenhang auch das, was die Schweizerische Akademie der Medizinischen Wissenschaften SAMW zum Thema publiziert hat: «Immer wieder gilt es in der Medizin die Frage zu stellen, ob eine Massnahme nötig und adäquat ist. So gesehen hat der Psychiater Eugen Bleuler das Thema einer evidenzbasierten Medizin schon vor 100 Jahren behandelt.» Jetzt denkt Mechtild Willi an ihren Grossvater und den Vater, die sie gepflegt hat. "Auch am Ende eines Lebens müssen wir uns fragen, was angemessen und was überflüssig ist. Alles entwickelt sich. Und wenn eine Krankheit und so eine Einschränkung kommt, entwickelt sich das Leben damit neu. Stets gilt es neu zu definieren: Was sind die Qualitäten, die ich brauche? Was macht mein Leben lebenswert?» Im Übrigen werde die Machbarkeit immer wieder überschätzt. "1982, als ich in der Psychiatrie arbeitete, gingen die Wissenschaftler davon aus, dass eine Depression demnächst behandelt oder gar geheilt werden kann, mit Hormonen oder wie auch immer. Und heute? Wo stehen wir diesbezüglich?»

\section{Apolitische Pflegende}

Das kritische Hinterfragen ist Thema auch in einer Masterarbeit, die Willi geschrieben hat. Hier geht es um die Bildungsreform im Gesundheitswesen und um Thesen aus der Praxis einer Pflegenden, die zur Pflegemanagerin geworden ist. Es sei schwierig, Pflegefachleute mit politischen Themen zu fordern, heisst es hier beispielsweise. «Ja», erläutert Willi, 


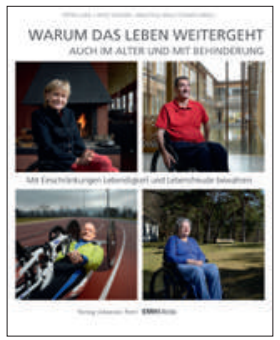

Peter Lude, Fritz Vischer, Mechtild Willi Studer (Hrsg.)

Warum das Leben weitergeht

Auch im Alter und mit Behinderung

Mit Einschränkungen Lebendigkeit

und Lebensfreude bewahren

Basel: Verlag Johannes Petri; 2014.

279 Seiten, 15 Abb. 45 CHF.

ISBN 978-3-03784-045-O

«Pflegende repräsentieren zwar eine sehr wichtige Berufsgruppe, leider aber sind sie eher apolitisch, haben wenig Biss. Zu sehr sind sie in der Regel mit dem Elementaren beschäftigt. Dabei wäre es wichtig, dass sie sich manifestieren und Einfluss nehmen würden.» Als Beispiel nennt sie die Abstimmung über die Mutterschaftsversicherung 2004. "In der Kaffeepause auf der Gebärabteilung fragte ich: Seid ihr zufrieden mit dem Abstimmungsresultat, Frauen? Niemand kannte den Zusammenhang meiner Frage, auch zum eigenen Beruf nicht.»

Arbeiten heisse nicht bloss eine Tätigkeitsliste abspulen, sondern auch gestalten und hinterfragen, das fördere sie auch bei ihren rund 400 Mitarbeitenden in Nottwil. «Der Pflegeberuf ist kreativ, sogar am Patientenbett ist eine spontane Programmänderung vielfach möglich, auch in einem hierarchisch strukturierten Betrieb. Wir müssen stets im Diskurs sein, mit unseren Patientinnen und Patienten, den Vorgesetzten und Ärzten.» Es gehe um tägliche Verrichtungen, den eigenen Berufsstand, aber auch um den grösseren gesundheitspolitischen Zusammenhang. Bloss: "Oft fehlt die Zivilcourage, der Wille, sich einzumischen, die Lust am Diskurs.»

Dazu komme etwas ganz anderes. Oft fehle, gerade für den Dialog mit der Ärzteschaft, die Basis für die Kommunikation: «Einige Ärzte sprechen heutzutage nicht einmal mehr Deutsch.»

\section{Feilschen um Geld}

Natürlich müssten Verbesserungsvorschläge von guten Argumenten begleitet sein, fährt sie fort, und illustriert das Gesagte gleich wieder mit einem eigenen Beispiel. «Mit Engagement und Stolz haben wir in Nottwil den Ansatz der Peergroups weiterentwickelt. Das erfolgreiche Modell, dass Behinderte von Behinderten wohl am meisten lernen können, wollten wir auf die Angehörigen ausdehnen. Sie leiden oft mehr als die direkt Betroffenen.» Weil die Coaches aber nicht ehrenamtlich arbeiteten und eine halbe Stelle hätte geschaffen werden müssen, liess man den inno- vativen Ansatz im Keim ersticken: «Das erfolgversprechende Projekt wird aus Spargründen nicht weiterverfolgt. Das macht mich traurig.» Endloses Feilschen um Geldbeträge hinter dem Komma würden viel wichtigere Diskussionen um Qualitätsstandards allzu oft verdrängen. «Beim Investitionsbudget werden Beträge für IT oder teure Apparate in der Regel abgenickt, Bereiche, die quantitativ schwierig messbar sind, aber direkt den Patienten zugute kommen, geben dagegen unproportional viel zu reden. Das macht mich zunehmend müde. Ich würde es begrüssen, wenn ein bestimmter Prozentsatz eines Investionsbudgets reserviert würde, um Dienstleistungen für Patienten innovativ zu verbessern.»

\section{Auftanken in der Auszeit}

Das Sabbatical soll dazu beitragen, für die letzte berufliche Etappe Energie zu tanken. Und, wie jeweils beim Jahreswechsel, neue Vorsätze zu fassen: «Ich will künftig noch besser abschätzen, wo sich ein Aufwand lohnt und wo nicht. Ich will mich nicht mehr immer für alles verantwortlich fühlen. Und ich will noch mehr mit mir selber und den anderen im Dialog sein.»

Eben ist Mechtild Willi von einer Südafrika-Reise zurückgekehrt, vorher genoss sie einen dreimonatigen Aufenthalt bei einer Bildhauerin in England. Zeit auch zum Reflektieren, wie gesagt. "Als junge Oberschwester habe ich erlebt, wie Pfleger jeden vergangenen Arbeitstag auf einer Liste abgestrichen haben, um zu sehen, wie viele Tage sie bis zur Pensionierung noch abarbeiten mussten. Das fand ich damals schon entwürdigend. Ich will meine verbleibenden Berufsjahre nicht aussitzen und zu einer zermürbenden Zeit werden lassen, sondern mal mit Zufriedenheit und einer positiven beruflichen Bilanz aufhören.» Die Grundlage ist gelegt. Die Adventszeit hat sie zum ersten Mal seit rund 40 Jahren - der Familie gewidmet. Im Januar hat sie sich mit zwei Hospitationen in anderen Spitälern wieder langsam an den beruflichen Alltag gewöhnt. Und Anfang Februar beginnt Mechtild Willi am alten Ort die neue Etappe im neuen Jahr, «inspiriert und erfrischt», wie sie sagt.

\section{Die nächste "Begegnung mit ...»}

Am Ende jeden Monats stellt die Schweizerische Ärztezeitung eine Persönlichkeit vor, die sich im Gesundheitswesen engagiert. Im Februar schildert Daniel Lüthi seine Begegnung mit Michele Genoni, Chefarzt der Klinik für Herzchirurgie am Stadtspital Triemli, Zürich. 